\title{
Multipartite entanglement in four-qubit cluster-class states
}

\author{
Yan-Kui Bai and Z. D. Wang* \\ Department of Physics and Center of Theoretical and Computational Physics, University of Hong Kong, Pokfulam Road, \\ Hong Kong, China
}

(Received 12 October 2007; published 7 March 2008)

\begin{abstract}
Based on quantitative complementarity relations (QCRs), we analyze the multipartite correlations in fourqubit cluster-class states. It is proven analytically that the average multipartite correlation $E_{m s}$ is entanglement monotone. Moreover, it is also shown that the mixed three-tangle is a correlation measure compatible with the QCRs in this kind of quantum state. More arrestingly, with the aid of the QCRs, a set of hierarchy entanglement measures is obtained rigorously in the present system.
\end{abstract}

DOI: 10.1103/PhysRevA.77.032313

PACS number(s): 03.67.Mn, 03.65.Ud, 03.65.Ta

\section{INTRODUCTION}

Entanglement, first noted by Einstein and Schrödinger, is one of the most important features of a many-body quantum system. Nowadays, it is a crucial physical resource widely used in quantum-information processing (QIP), as in quantum communication $[1,2]$ and quantum computation [3-5]. Therefore, the characterization of entanglement, especially at a quantitative level, is fundamentally important. Compared with bipartite entanglement, which is now well understood in many aspects, the characterization of multipartite entanglement is still very challenging though a lot of effort has been made (see [6]).

It is widely accepted that a good entanglement measure should be non-negative, invariant under local unitary (LU) transformation, and nonincreasing on average under local operations and classical communications (LOCC), i.e., entanglement monotone [7]. Recently, based on quantitative complementarity relations (QCRs) [8], an average multipartite correlation measure $E_{m s}$ is introduced, which was proved to satisfy the first two conditions [9]. From much numerical analysis, it was conjectured that $E_{m s}$ also has the entanglement monotone property and thus may be able to characterize the multipartite entanglement in a four-qubit pure state [9]. However, the analytical proof of the conjecture is extremely difficult for a general quantum state. In this sense, it seems helpful to look into the conjecture in certain cases, which, on one hand, allows us to obtain exact results, and, on the other hand, gives us useful information beyond bipartite entanglement.

Cluster states, which are typically multipartite entangled states, are utilized in quantum error-correcting codes [10] and tests of quantum nonlocality [11]. Moreover, they are also a universal resource in one-way quantum computation [4]. In optical systems, a four-qubit cluster state has been prepared and applied to the Grover search algorithm [12,13]. More recently, a six-photon cluster state was also produced [14]. So, in order to make better use of the cluster state, it is quite desirable to explore quantitatively the entanglement in this kind of system.

In this paper, we analyze the multipartite quantum correlations in four-qubit cluster-class states. Here, by a cluster-

\footnotetext{
*zwang@hkucc.hku.hk
}

class state, we mean the output state of a cluster state under stochastic LOCC (SLOCC $[15,16])$. For this class of quantum states, we prove exactly that the average multipartite correlation $E_{m s}$ is entanglement monotone. Moreover, it is shown that the three- and four-qubit correlations $t_{3}$ and $t_{4}$ are also entanglement monotone when setting $t_{3}$ to be a mixed three-tangle. More intriguingly, a set of hierarchy entanglement measures are thus obtained rigorously in the system. The paper is organized as follows. In Sec. II, the entanglement monotone property of multipartite correlations in the cluster-class states is proven exactly. In Sec. III, we address several relevant key issues and give a brief conclusion.

\section{MULTIPARTITE QUANTUM CORRELATIONS IN FOUR-QUBIT CLUSTER-CLASS STATES}

Before analyzing these quantum correlations, we first recall the QCRs and the definition of average multipartite quantum correlation. As an essential principle of quantum mechanics, complementarity often refers to mutually exclusive properties. The quantitative version of the complementarity relation in an $N$-qubit pure state is also provided and formulated as [8] $\tau_{k\left(R_{k}\right)}+S_{k}^{2}=1$, where the linear entropy $\tau_{k\left(R_{k}\right)}$ characterizes the total quantum correlation of qubit $k$ with the remaining qubits $R_{k}$ and $S_{k}^{2}$ is a measure of single-particle properties. For an $\mathrm{N}$-qubit pure state, the linear entropy is contributed by the different levels of quantum correlation, i.e., $\left\{t_{2}, t_{3}, \ldots, t_{N}\right\}$, in which $t_{m}$ represents the genuine $m$-qubit correlation for $m=2,3, \ldots, N[9,17]$. Based on the QCRs, an average multipartite correlation measure in a fourqubit pure state is introduced [9]:

$$
E_{m s}\left(\Psi_{4}\right)=\frac{M}{4}=\frac{M_{A}+M_{B}+M_{C}+M_{D}}{4},
$$

where $M$ is the sum of the single residual correlations and $M_{k}$ is defined as $M_{k}=\tau_{k\left(R_{k}\right)}-\Sigma_{l \in R_{k}} C_{k l}^{2}$ (here, the square of the concurrence quantifies the two-qubit correlation). It is conjectured that $E_{m s}$ is entanglement monotone and can characterize the multipartite entanglement in the system. However, the proof of this property is extremely difficult for a generic quantum state, although a numerical analysis supports the conjecture.

Due to the important applications in QIP, cluster states have been paid more and more attention in recent years. As 


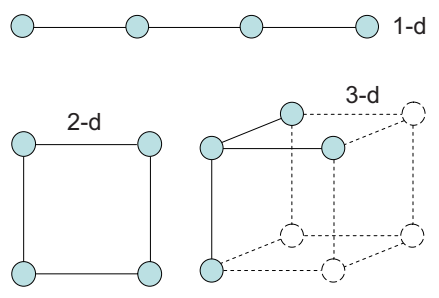

FIG. 1. (Color online) Schematic graphs of four-qubit cluster states in $1 \mathrm{D}, 2 \mathrm{D}$, and $3 \mathrm{D}$ lattices.

shown in Fig. 1, these states are associated with graphs where each vertex represents a qubit prepared in the initial state $(|0\rangle+|1\rangle) / \sqrt{2}$ and each edge represents a controlled phase gate applied between two qubits [4]. In this paper, we will consider the multipartite quantum correlations in fourqubit cluster-class states that are related to the cluster states by SLOCC. In the following, we will analyze the entanglement monotone property of the average multipartite correlation $E_{m s}$ and the three- and four-qubit correlations $t_{3}$ and $t_{4}$ in this class of quantum state.

\section{A. Average multipartite quantum correlation and entanglement monotone}

In one-dimensional (1D) lattices, the four-qubit cluster state can be written as $\left|\mathcal{C}_{4}^{(1)}\right\rangle=(|0000\rangle+|0011\rangle+|1100\rangle$ $-|1111\rangle) / 2$ after LU transformation. The entanglement monotone property requires that the correlation $E_{m s}$ does not increase on average under LOCC. It is known that any local operation can be implemented by a sequence of two-outcome positive operator-valued measures (POVMs) such as $\left\{A_{1}, A_{2}\right\}$ which satisfies $A_{1}^{\dagger} A_{1}+A_{2}^{\dagger} A_{2}=I$ [16]. According to the singular-value decomposition [16], the POVM operators can be written as $A_{1}=U_{1} \operatorname{diag}\{\alpha, \beta\} V$ and $A_{2}$ $=U_{2} \operatorname{diag}\left\{\sqrt{1-\alpha^{2}}, \sqrt{1-\beta^{2}}\right\} V$, respectively, where $U_{i}$ and $V$ are unitary matrices, and $\alpha$ and $\beta$ are real numbers in the range $(0,1)$. Due to the LU invariance of the $E_{m s}$, we need only consider the diagonal matrices. The output state of $\left|\mathcal{C}_{4}^{(1)}\right\rangle$ under a general POVM operator (i.e., the SLOCC operation) has the form

$$
\left|\Psi^{(1)}\right\rangle=a|0000\rangle+b|0011\rangle+c|1100\rangle-d|1111\rangle,
$$

where the normalized parameters $a, b, c$, and $d$ are complex numbers and we refer to $\left|\Psi^{(1)}\right\rangle$ as the cluster-class state [18]. Furthermore, since the form of this quantum state is not changed under the next POVM, the entanglement monotone property of $E_{m s}\left(\Psi^{(1)}\right)$ will be satisfied only if the quantity is nonincreasing under the first level of the POVM.

For the quantum state $\left|\Psi^{(1)}\right\rangle$, the two-qubit reduced density matrix of subsystem $A B$ reads

$$
\rho_{A B}=\left(\begin{array}{cccc}
|a|^{2}+|b|^{2} & 0 & 0 & a c^{*}-b d^{*} \\
0 & 0 & 0 & 0 \\
0 & 0 & 0 & 0 \\
a^{*} c-b^{*} d & 0 & 0 & |c|^{2}+|d|^{2}
\end{array}\right) .
$$

Note that the two-qubit quantum correlation may be defined as $t_{2}\left(\rho_{A B}\right)=C^{2}\left(\rho_{A B}\right)$, where the concurrence $C\left(\rho_{A B}\right)$
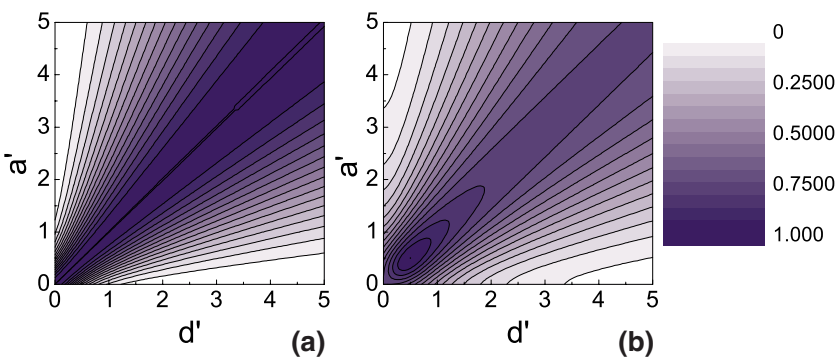

FIG. 2. (Color online) Contour plots of the average multipartite entanglement $E_{m s}$ in four-qubit cluster-class states $\left|\Psi^{(1)}\right\rangle$ and $\left|\Psi^{(2)}\right\rangle$, where the non-normalized parameters $a^{\prime}$ and $d^{\prime}$ are in the range $[0,5]$ and the parameters $b^{\prime}=c^{\prime}=0.5$ are fixed.

$=\max \left[0,\left(\sqrt{\lambda_{1}}-\sqrt{\lambda_{2}}-\sqrt{\lambda_{3}}-\sqrt{\lambda_{4}}\right)\right]$ with the decreasing positive real numbers $\lambda_{i}$ being the eigenvalues of the matrix $\rho_{A B}\left(\sigma_{y}\right.$ $\left.\otimes \sigma_{y}\right) \rho_{A B}^{*}\left(\sigma_{y} \otimes \sigma_{y}\right)$ [19]. After a simple calculation, we get $C_{A B}=2\left|a^{*} c-b^{*} d\right|$. Similarly, we have $C\left(\rho_{C D}\right)=2\left|a^{*} b-c^{*} d\right|$ and $C\left(\rho_{i j}\right)=0$ for other subsystems. The linear entropy of qubit $A, \tau_{A\left(R_{A}\right)}\left(=4 \operatorname{det} \rho_{A}\right)$ [20], can quantify the total quantum correlation between subsystems $A$ and $B C D$. So, the multipartite correlation related to qubit $A$, i.e., the residual correlation, is

$$
M_{A}\left(\Psi^{(1)}\right)=\tau_{A\left(R_{A}\right)}-C_{A B}^{2}=4|a d+b c|^{2} .
$$

With a similar derivation, we can obtain $M_{B}=M_{C}=M_{D}$ $=M_{A}$, which means that the single residual correlation $M_{k}\left(\Psi^{(1)}\right)$ is invariant under permutations of qubits and the average correlation $E_{m s}\left(\Psi^{(1)}\right)=M_{A}\left(\Psi^{(1)}\right)$.

Under the POVM $\left\{A_{1}, A_{2}\right\}$ performed on the subsystem $A$, two quantum states $\left|\Phi_{1}^{(1)}\right\rangle=A_{1}\left|\Psi^{(1)}\right\rangle / \sqrt{p_{1}}$ and $\left|\Phi_{2}^{(1)}\right\rangle$ $=A_{2}\left|\Psi^{(1)}\right\rangle / \sqrt{p_{2}}$ are available with probabilities $p_{i}$ $=\operatorname{tr}\left(A_{i}\left|\Psi^{(1)}\right\rangle\left\langle\Psi^{(1)}\right| A_{i}^{\dagger}\right)$ for $i=1,2$. Note that the linear entropy and the concurrence are invariant under determinant one SLOCC operation [i.e., for the quantum states $\left|\Psi^{(1)}\right\rangle,\left|\Phi_{1}^{(1)}\right\rangle$, and $\left|\Phi_{2}^{(1)}\right\rangle$, the two measures are invariant if the POVM operator satisfies $\left.\operatorname{det}\left(A_{i}\right)=1\right][21]$; we can obtain $M_{A}\left(\Phi_{1}^{(1)}\right)=\left(\alpha^{2} \beta^{2} / p_{1}^{2}\right) M_{A}\left(\Psi^{(1)}\right) \quad$ and $\quad M_{A}\left(\Phi_{1}^{(2)}\right)=\left[\left(1-\alpha^{2}\right)(1\right.$ $\left.-\beta^{2}\right) /$

$\left.p_{2}^{2}\right] M_{A}\left(\Psi^{(1)}\right)$. With a similar deduction as that in Ref. [16], we can derive the following relation:

$$
M_{A}\left(\Psi^{(1)}\right)-p_{1} M_{A}\left(\Phi_{1}^{(1)}\right)-p_{2} M_{A}\left(\Phi_{2}^{(1)}\right) \geq 0 .
$$

Combining the permutation invariance of the $M_{k}\left(\Psi^{(1)}\right)$, we can draw the conclusion that the single residual correlation $M_{A}\left(\Psi^{(1)}\right)=E_{m s}\left(\Psi^{(1)}\right)$ is entanglement monotone and can characterize the multipartite entanglement in the system.

For this kind of quantum state, the contour plot of $E_{m s}$ versus the non-normalized real parameters $a^{\prime}$ and $d^{\prime}$ is depicted in Fig. 2(a), where the parameters $b^{\prime}=c^{\prime}=0.5$ are fixed. In the regions near $\left(a^{\prime}=d^{\prime}=0\right)$ and $\left(a^{\prime}, d^{\prime} \gg 0.5\right)$, the multipartite entanglement has larger values, as the quantum $a^{\prime}$ state $\left|\Psi^{(1)}\right\rangle$ tends to the Greenberger-Horne-Zeilinger $(\mathrm{GHZ})$ state. In the regions $\left(a^{\prime} \gg b^{\prime}, c^{\prime}, d^{\prime}\right)$ and $\left(d^{\prime}\right.$ $\left.\gg a^{\prime}, b^{\prime}, c^{\prime}\right), E_{m s}$ has smaller values, as the quantum state approaches the product state. In particular, when the real 
parameters and $b^{\prime}=c^{\prime}$, the multipartite entanglement reaches the maximum $E_{m s}=1$. In this case, the quantum state can be rewritten as

$$
\left|\Pi_{4}\right\rangle=\left(|00\rangle \otimes|\varphi\rangle+|11\rangle \otimes\left|\varphi^{\perp}\right\rangle\right) / \sqrt{2}
$$

where $|\varphi\rangle=\left(a^{\prime}|00\rangle+b^{\prime}|11\rangle\right) / \sqrt{a^{\prime 2}+b^{\prime 2}}$ and $\left|\varphi^{\perp}\right\rangle=b^{\prime}|00\rangle$ $-a^{\prime}|11\rangle / \sqrt{a^{\prime 2}+b^{\prime 2}}$. This state is the generalized Bell state, i.e., the maximal bipartite entangled state between subsystems $A B$ and $C D$. When $|\varphi\rangle$ is a product state, $\left|\Pi_{4}\right\rangle$ is a GHZ state. When $|\varphi\rangle$ is a Bell state, $\left|\Pi_{4}\right\rangle$ is a cluster state $\left|\mathcal{C}_{4}^{(1)}\right\rangle$.

In two-dimensional lattices, the four-qubit cluster-class state has the form

$$
\left|\Psi^{(2)}\right\rangle=a|0000\rangle-b|0111\rangle-c|1010\rangle+d|1101\rangle,
$$

where the parameters $a, b, c$, and $d$ are also complex. This kind of quantum state is related to the box cluster state $\left[\left|\mathcal{C}_{4}^{(2)}\right\rangle=(|0000\rangle-|0111\rangle-|1010\rangle+|1101\rangle) / 2\right] \quad$ via SLOCC. For the cluster-class state, we can obtain the concurrences $C_{A C}^{2}=4(|a c|-|b d|)^{2}$ and $C_{i j}^{2}=0$ for the other subsystems. Unlike in the $1 \mathrm{D}$ case, the single residual correlation $M_{k}\left(\Psi^{(2)}\right)$ is not permutation invariant and does not satisfy the entanglement monotone property. As an example, we consider the quantum state $\left|\Psi^{(2)}\right\rangle$, where the non-normalized coefficients $a^{\prime}=b^{\prime}=2, c^{\prime}=0.2$, and $d^{\prime}=3$. After a simple calculation, we have $M_{A}=0.5643$ and $M_{C}=0.2915$. Under the POVM performed on qubit $A$ (here $\alpha=0.9$ and $\beta=0.2$ ), the change of the residual correlation is $\Delta M_{C}=M_{C}\left(\Psi^{(2)}\right)$ $-p_{1} M_{C}\left(\Phi_{1}\right)-p_{2} M_{C}\left(\Phi_{2}\right)=-0.1151$.

However, the average multipartite correlation

$$
E_{m s}\left(\Psi^{(2)}\right)=3\left(|a|^{2}+|c|^{2}\right)\left(|b|^{2}+|d|^{2}\right)+4|a b c d|
$$

is entanglement monotone, which can be proven as follows. First, we consider the POVM $\left\{A_{1}, A_{2}\right\}$ performed on the subsystem $A$. Due to the LU-invariant property of the $E_{m s}$, we need only consider the diagonal matrices in the singularvalue decomposition form, as the output states $\left|\Phi_{1}\right\rangle$ and $\left|\Phi_{2}\right\rangle$ are obtained with the probabilities $p_{1}$ and $p_{2}$, respectively. The correlation $E_{m s}\left(\Psi^{(2)}\right)$ can be separated into two components $\zeta_{1}=\left(\tau_{A\left(R_{A}\right)}-2 C_{A C}^{2}\right) / 4$ and $\zeta_{2}=\left(\tau_{B\left(R_{B}\right)}+\tau_{C\left(R_{C}\right)}\right.$ $\left.+\tau_{D\left(R_{D}\right)}\right) / 4$, on which the effects are different under the POVM. The component $\zeta_{1}$ is invariant under the determinant one SLOCC. With this property, we can derive

$$
\begin{aligned}
\Delta \zeta_{1} & =\zeta_{1}\left(\Psi^{(2)}\right)-p_{1} \zeta_{1}\left(\Phi_{1}\right)-p_{2} \zeta_{1}\left(\Phi_{2}\right) \\
& =\left[1-\alpha^{2} \beta^{2} / p_{1}-\left(1-\alpha^{2}\right)\left(1-\beta^{2}\right) / p_{2}\right] \zeta_{1}\left(\Psi^{(2)}\right),
\end{aligned}
$$

where $\zeta_{1}\left(\Psi^{(2)}\right)=(|a d|+|b c|)^{2}-(|a c|-|b d|)^{2}$ (in the general case, this quantity is not guaranteed to be non-negative). For the component $\zeta_{2}$, the change is $\Delta \zeta_{2}=\zeta_{2}\left(\Psi^{(2)}\right)-p_{1} \zeta_{2}\left(\Phi_{1}\right)$ $-p_{2} \zeta_{2}\left(\Phi_{2}\right)=\Sigma_{k \neq A}\left[\tau\left(\rho_{k}\right)-p_{1} \tau\left(\rho_{k}^{1}\right)-p_{2} \tau\left(\rho_{k}^{2}\right)\right]$, which is equivalent to the changes of the linear entropies induced by the mixed state decomposition of subsystems $\rho_{k}$ for $k=B, C, D$ [22]. After some tedious calculation, the change of the average multipartite correlation is

$$
\begin{aligned}
\Delta_{A} E_{m s}= & \Delta \zeta_{1}+\Delta \zeta_{2} \\
= & \left(\alpha^{2}-\beta^{2}\right)^{2}\left[4|a b c d|\left(|a|^{2}+|b|^{2}\right)\left(|c|^{2}+|d|^{2}\right)\right. \\
& \left.+3\left(|b c|^{2}-|a d|^{2}\right)^{2}\right] / p_{1} p_{2},
\end{aligned}
$$

which is obviously a non-negative number. This means that the correlation $E_{m s}\left(\Psi^{(2)}\right)$ does not increase on average under the POVM performed on qubit $A$. But, since the quantities $\zeta_{1}$ and $\zeta_{2}$ are variant under the permutation of two qubits, we still need to consider the POVMs performed on the subsystems $B, C$, and $D$. After a similar analysis, we can derive the change of the correlation under the POVM on qubit $C$ as $\Delta_{C} E_{m s}=\left(\alpha^{2}-\beta^{2}\right)^{2}\left[4|a b c d|\left(|a|^{2}+|d|^{2}\right)\left(|b|^{2}+|c|^{2}\right)+3\left(|a b|^{2}\right.\right.$ $\left.\left.-|c d|^{2}\right)^{2}\right] / p_{1} p_{2}$, which is also non-negative. For the POVM on the subsystem $B$, one can separate the correlation $E_{m s}$ into two components $\kappa_{1}=\left(\tau_{B\left(R_{B}\right)}\right) / 4$ and $\kappa_{2}=\left(\sum_{k \neq B} \tau_{k\left(R_{k}\right)}\right.$ $-2 C_{A C}^{2}$ ) /4 (the non-negative property of $\kappa_{2}$ is guaranteed by the monogamy relation [23]). $\kappa_{1}$ is nonincreasing due to the SLOCC invariance, and $\kappa_{2}$ is nonincreasing because of the concave and convex properties of the linear entropy and the concurrence, respectively. Therefore, $E_{m s}$ is also nonincreasing under this POVM. The case of the POVM on the subsystem $D$ is similar. According to the above analysis, we can draw the conclusion that the correlation $E_{m s}\left(\Psi^{(2)}\right)$ is entanglement monotone and can characterize the multipartite entanglement in the system.

In Fig. 2(b), the change of $E_{m s}\left(\Psi^{(2)}\right)$ with the nonnormalized real parameters $a^{\prime}$ and $d^{\prime}\left(b^{\prime}=c^{\prime}=0.5\right.$ are fixed) is plotted. When $\left(a^{\prime} \gg b^{\prime}, c^{\prime}, d^{\prime}\right)$ and $\left(d^{\prime} \gg a^{\prime}, b^{\prime}, c^{\prime}\right), E_{m s}$ $\approx 0$ and the quantum states tend to the four-qubit product state. When $\left(a^{\prime}, d^{\prime} \approx 0\right)$ and $\left(a^{\prime}, d^{\prime} \gg b^{\prime}, c^{\prime}\right)$, the multipartite entanglement has rather large values $\left(E_{m s} \approx 0.75\right)$, where the quantum state approximates to the product state of a singlequbit state and a three-qubit GHZ state. The maximum $E_{m s}$ $=1$ appears at the point $a^{\prime}=d^{\prime}=0.5$, where the quantum state is just the box cluster state $\left|\mathcal{C}_{4}^{(2)}\right\rangle$.

Finally, we address the entanglement monotone property of $E_{m s}$ in a three-dimensional cluster-class state, which is a trivial case. This state has the form

$$
\left|\Psi^{(3)}\right\rangle=a|0000\rangle+b|1111\rangle,
$$

and relates to the four-qubit Greenberger-Horne-Zeilinger state via the SLOCC operation. The quantum state $\left|\Psi^{(3)}\right\rangle$ is invariant under the permutation of qubits and all its twoqubit concurrences are zeros. Under the next level of the POVM, the same properties still hold. So the single residual correlation $M_{k}=\tau_{k\left(R_{k}\right)}$ is entanglement monotone and satisfies $M_{A}=M_{B}=M_{C}=M_{D}$. It is obvious that the average correlation $E_{m s}\left(\Psi^{(3)}\right)=M_{k}=4|a b|^{2}$ is also entanglement monotone and can characterize the multipartite entanglement in the system.

\section{B. Three- and four-qubit entanglement measures}

In a four-qubit pure state $|\Psi\rangle_{A B C D}$, there are five multipartite correlation parameters (cf. the Venn diagram in [9]), i.e., one genuine four-qubit correlation $t_{4}\left(|\Psi\rangle_{A B C D}\right)$ and four three-qubit correlations $t_{3}\left(\rho_{i j k}\right)$. According to the QCRs, we have a set of equations [9] 


$$
t_{4}(|\Psi\rangle)+\sum_{i<j \neq k} t_{3}\left(\rho_{i j k}\right)=M_{k},
$$

where $M_{k}$ is the single-residual correlation related to qubit $k$, and the subscripts $i, j, k=A, B, C, D$. Note that these four equations are unable to determine completely the five correlation parameters. In fact, at least one additional independent relation for either $t_{3}$ or $t_{4}$ is needed in this case.

As is known, the mixed three-tangle is a good entanglement measure for a three-qubit mixed state; it is defined as [24]

$$
\tau_{3}\left(\rho_{i j k}\right)=\min \sum_{\left\{p_{x}, \phi_{x}\right\}} p_{x} \tau\left(\phi_{x}\right)
$$

where $\tau$ is the pure state three-tangle [25] and the minimum runs over all the pure state decompositions of $\rho_{i j k}$. However, it is shown in Ref. [9] that $\tau_{3}$ is not compatible with the QCRs in some specific four-qubit pure states [for example, the quantum state $|\psi\rangle_{A B C D}=(|0000\rangle+|1011\rangle+|1101\rangle$ $+|1110\rangle) / 2$ [26]]. So, for the cluster-class states, it is necessary to check whether or not the $\tau_{3}$ can quantify correctly the $t_{3}$ in the QCRs. If $\tau_{3}$ does this, we are able to obtain the genuine four-qubit correlation $t_{4}$ in terms of Eq. (11).

For the cluster-class state $\left|\Psi^{(1)}\right\rangle$ in 1D lattices, the threequbit reduced density matrices have the form $\rho_{i j k}=p_{1}|0\rangle\left\langle\left. 0\right|_{i}\right.$ $\otimes|\phi\rangle\left\langle\left.\phi\right|_{j k}+p_{2} \mid 1\right\rangle\left\langle\left. 1\right|_{i} \otimes \mid \psi\right\rangle\left\langle\left.\psi\right|_{j k}\right.$, in which $\left.\mid \phi\right\rangle$ and $|\psi\rangle$ are twoqubit entangled states. If one uses the mixed three-tangle to quantify the three-qubit correlation, the relation $t_{3}\left(\rho_{i j k}\right)$ $=\tau_{3}\left(\rho_{i j k}\right)=0$ can be obtained. Substituting this relation into Eq. (11), one can solve the genuine four-qubit correlation $t_{4}=M_{k}=4|a d+b c|^{2}$. According to the analysis in Sec. II A, we know that the quantity $\tau_{4}=t_{4}$ satisfies all three requirements of an entanglement measure. Therefore, for the cluster-class state $\left|\Psi^{(1)}\right\rangle$, a set of correlation measures $\left\{\tau_{2}, \tau_{3}, \tau_{4}\right\}$ which all are entanglement monotone [we define $\left.\tau_{2}\left(\rho_{i j}\right)=C_{i j}^{2}\right]$ can characterize the genuine two-, three-, and four-qubit entanglement in the system. For the cluster-state $\left|\Psi^{(3)}\right\rangle$ in $3 \mathrm{D}$ lattices, the case is similar. Its three-qubit reduced density matrix is $\rho_{i j k}=|a|^{2}|000\rangle\left\langle\left. 000|+| b\right|^{2} \mid 111\right\rangle\langle 111|$ and the corresponding three-tangle $\tau_{3}$ is zero. After using $\tau_{3}$ to quantify the correlation $t_{3}$, one can solve the correlation $t_{4}=\tau_{4}=M_{k}=4|a b|^{2}$, which is also entanglement monotone. So the correlation measures $\left\{\tau_{2}, \tau_{3}, \tau_{4}\right\}$ can characterize the different levels of entanglement in the cluster-class state $\left|\Psi^{(3)}\right\rangle$.

In the cluster-class state $\left|\Psi^{(2)}\right\rangle$, the situation is nontrivial. If one uses the mixed three-tangle to quantify the correlation $t_{3}$, it is straightforward to find that $\tau_{3}\left(\rho_{A B C}\right)=0$ and $\tau_{3}\left(\rho_{A C D}\right)=0$. Substituting the two zero $t_{3}$ 's into Eq. (11), one can obtain the other three multipartite correlations $t_{4}\left(\Psi^{(2)}\right)$ $=16|a b c d|, \quad t_{3}\left(\rho_{A B D}\right)=4(|a d|-|b c|)^{2}, \quad$ and $t_{3}\left(\rho_{B C D}\right)=4(|a b|$ $-|c d|)^{2}$. At this stage, we need to consider whether or not the mixed three-tangle $\tau_{3}$ is compatible with the QCRs in this system and whether the correlation $t_{4}$ is appropriate to characterize the genuine four-qubit entanglement.

We first analyze the compatibility of $\tau_{3}$ with the QCRs in the system. The decomposition of $\rho_{A B D}$ into its eigenstates can be written as

$$
\rho_{A B D}=p\left|\psi_{1}\right\rangle\left\langle\psi_{1}|+(1-p)| \psi_{2}\right\rangle\left\langle\psi_{2}\right|,
$$

where

$$
\left|\psi_{1}\right\rangle=(a|000\rangle+d|111\rangle) / \sqrt{p} \text {, }
$$

$\left|\psi_{2}\right\rangle=(b|011\rangle$ $+c|100\rangle) / \sqrt{1-p}$, and $p=|a|^{2}+|d|^{2}$. It is well known that any other decomposition can be obtained with a unitary transformation on the eigenvectors [27]. Hence, the vectors of any decomposition of $\rho_{A B D}$ are linear combination of $\left|\psi_{1}\right\rangle$ and $\left|\psi_{2}\right\rangle$, i.e.,

$$
\begin{aligned}
|Z(q, \phi)\rangle & =\sqrt{q}\left|\psi_{1}\right\rangle-e^{i \phi} \sqrt{1-q}\left|\psi_{2}\right\rangle \\
& =\widetilde{a}|000\rangle-e^{i \phi} \widetilde{b}|011\rangle-e^{i \phi} \widetilde{c}|100\rangle+\widetilde{d}|111\rangle,
\end{aligned}
$$

where $\tilde{a}=a \gamma, \tilde{b}=b \eta, \tilde{c}=c \eta$, and $\tilde{d}=d \gamma$, with $\gamma=\sqrt{q / p}$ and $\eta=\sqrt{(1-q) /(1-p)}$. For this pure state, the reduced density matrix of qubits $A B$ is

$$
\rho_{A B}(Z)=\left(\begin{array}{cccc}
|\widetilde{a}|^{2} & 0 & -\widetilde{a} \widetilde{c}^{*} e^{-i \phi} & 0 \\
0 & |\widetilde{b}|^{2} & 0 & -\widetilde{b} \widetilde{d}^{*} e^{i \phi} \\
-\widetilde{a}^{*} \tilde{c} e^{i \phi} & 0 & |\widetilde{c}|^{2} & 0 \\
0 & -\widetilde{b}^{*} \widetilde{d} e^{-i \phi} & 0 & |\widetilde{d}|^{2}
\end{array}\right)
$$

and its concurrence is zero (in fact, $\rho_{A B}$ is a mix of two product states). Similarly, for the quantum state $\rho_{A D}(Z)$, we can obtain $C_{A D}=0$ as well. So, in any pure state decomposition of $\rho_{A B D}$, the entanglements of subsystems $A B$ and $A D$ are both zeros. Then, according to the definition of the mixed state three-tangle, we have the following relation:

$$
\begin{aligned}
\tau_{3}\left(\rho_{A B D}\right) & =\min \sum_{\left\{p_{x}, Z_{x}\right\}} p_{x} \tau\left[Z_{x}(q, \phi)\right] \\
& =\min \sum_{\left\{p_{x}, Z_{x}\right\}} p_{x}\left[\tau_{A\left(R_{A}\right)}^{(x)}-\left(C_{A B}^{(x)}\right)^{2}-\left(C_{A D}^{(x)}\right)^{2}\right] \\
& =\min \sum_{\left\{p_{x}, Z_{x}\right\}} p_{x} \tau_{A\left(R_{A}\right)}^{(x)} \\
& =C_{A: B D}^{2}\left(\rho_{A B D}\right) \\
& =4(|a d|-|b c|)^{2}
\end{aligned}
$$

where we have replaced the basis $\{|00\rangle,|11\rangle\}_{B D}$ with $\{|\widetilde{0}\rangle,|\widetilde{1}\rangle\}_{B D}$ for the calculation of the last equation. This value coincides with the correlation $t_{3}\left(\rho_{A B D}\right)$ obtained using the QCRs. For the quantum state $\rho_{B C D}$, we can get $\tau_{3}\left(\rho_{B C D}\right)$ $=4(|a b|-|c d|)^{2}=t_{3}\left(\rho_{B C D}\right)$ after a similar derivation. Therefore, in the cluster-class state $\left|\Psi^{(2)}\right\rangle$, the mixed three-tangle $\tau_{3}$ can quantify correctly the correlation $t_{3}$ and is compatible with the QCRs.

With the QCRs, we solve the genuine four-qubit correlation $t_{4}\left(\Psi^{(2)}\right)=16|a b c d|$, which is obviously non-negative. The LU-invariant property is guaranteed by the corresponding property of the correlations $M_{k}$ and $t_{3}$ in Eq. (11). Before using $t_{4}\left(\Psi^{(2)}\right)$ to characterize the genuine four-qubit entanglement in the system, we should prove first that it is entanglement monotone. Since the correlation $t_{4}$ is invariant under the permutations of qubits, we need only consider the 

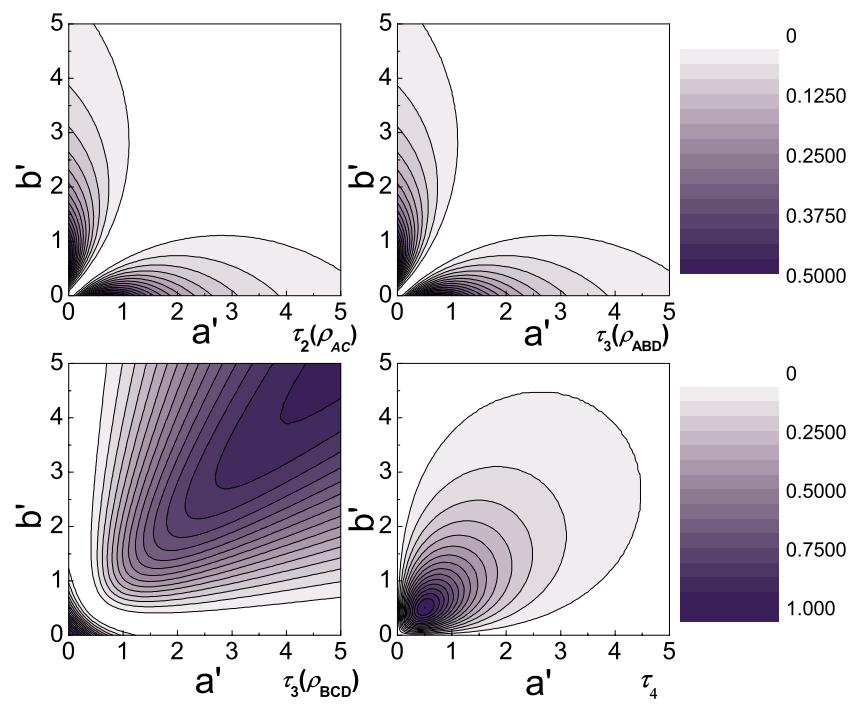

FIG. 3. (Color online) Two-, three-, and four-qubit entanglement measures versus the non-normalized real parameters $a^{\prime}$ and $b^{\prime}$ in the cluster-class state $\left|\Psi^{(2)}\right\rangle=a^{\prime}|0000\rangle-b^{\prime}|0111\rangle-0.5|1010\rangle$ $+0.5|1101\rangle$.

POVM $\left\{A_{1}, A_{2}\right\}$ performed on the subsystem $A$ in which the diagonal matrices are $\operatorname{diag}\{\alpha, \beta\}$ and $\operatorname{diag}\left\{\sqrt{1-\alpha^{2}}, \sqrt{1-\beta^{2}}\right\}$, respectively. After the POVM, two output states are available with probabilities $p_{1}$ and $p_{2}$, respectively, and the change of the correlation is $\Delta t_{4}\left(\Psi^{(2)}\right)=\left[1-\alpha^{2} \beta^{2} / p_{1}-\left(1-\alpha^{2}\right)(1\right.$ $\left.\left.-\beta^{2}\right) / p_{2}\right] t_{4}\left(\Psi^{(2)}\right)$. Due to the non-negativity of the two factors in $\Delta t_{4}[16]$, the correlation $t_{4}\left(\Psi^{(2)}\right)=\tau_{4}$ is entanglement monotone. Therefore, the set of correlation measures $\left\{\tau_{2}, \tau_{3}, \tau_{4}\right\}$ is able to characterize the entanglements of two, three, and four qubits in the cluster-class state $\left|\Psi^{(2)}\right\rangle$, namely, they can be good entanglement measures for the corresponding multibody systems.

In Fig. 3, the variations of the two-, three-, and four-qubit entanglements with the non-normalized parameters $a^{\prime}$ and $b^{\prime}$ are plotted. The behaviors of $C_{A C}^{2}$ and $\tau_{3}\left(\rho_{A B D}\right)$ are the same and both attain the maximum 0.4999 when $\left(a^{\prime}=0, b^{\prime}\right.$ $=0.7)$ and $\left(a^{\prime}=0.7, b^{\prime}=0\right)$. The value of $\tau_{3}\left(\rho_{B C D}\right)$ tends to 1 when $\left(a^{\prime}=b^{\prime} \approx 0\right)$ and $\left(a^{\prime}=b^{\prime} \gg 0.5\right)$, because the quantum state $\rho_{B C D}$ approximates the pure GHZ state in these regions. The genuine four-qubit entanglement $\tau_{4}$ will be 1 when $a^{\prime}$ $=b^{\prime}=0.5$. At this point, the quantum state is just the box cluster state $\left|\mathcal{C}_{4}^{(2)}\right\rangle$.

Based on the above analysis, we conclude that not only is the mixed three-tangle $\tau_{3}$ a compatible correlation measure with the QCRs but also a set of hierarchy measures $\left\{\tau_{2}, \tau_{3}, \tau_{4}\right\}$ can, respectively, quantify the two-, three-, and four-qubit entanglement in the cluster-class states, as listed in Table I.

\section{DISCUSSION AND CONCLUSION}

For the cluster-class state $\left|\Psi^{(2)}\right\rangle$, the single residual correlation $M_{C}$ is not entanglement monotone as we showed in Sec. II A. Here, we explain the reason. This residual correlation can be written as $M_{C}=\tau_{4}+\tau_{3}\left(\rho_{B C D}\right)$ in terms of the
TABLE I. Entanglement measures in different four-qubit cluster-class states.

\begin{tabular}{lccc}
\hline \hline State parameters & $\left|\Psi^{(1)}\right\rangle$ & $\left|\Psi^{(2)}\right\rangle$ & $\left|\Psi^{(3)}\right\rangle$ \\
\hline$\tau_{4}$ & $4|a d+b c|^{2}$ & $16|a b c d|$ & $4|a b|^{2}$ \\
$\tau_{3}\left(\rho_{A B D}\right)$ & 0 & $4(|a d|-|b c|)^{2}$ & 0 \\
$\tau_{3}\left(\rho_{B C D}\right)$ & 0 & $4(|a b|-|c d|)^{2}$ & 0 \\
$\tau_{2}\left(\rho_{A B}\right)$ & $4\left|a^{*} c-b^{*} d\right|^{2}$ & 0 & 0 \\
$\tau_{2}\left(\rho_{A C}\right)$ & 0 & $4(|a c|-|b d|)^{2}$ & 0 \\
$\tau_{2}\left(\rho_{C D}\right)$ & $4\left|a^{*} b-c^{*} d\right|^{2}$ & 0 & 0 \\
\hline \hline
\end{tabular}

analysis in Sec. II B. Although the two components are both entanglement monotone functions under the POVMs performed on the subsystems $B, C$, and $D$, the effects of the POVMs on the subsystem $A$ are different from them. Due to the invariance of the qubit permutations, $\tau_{4}$ is still monotone under this POVM. For the reduced density matrix $\rho_{B C D}$, the effect of the POVM on qubit $A$ is equivalent to a mixed state decomposition of $\rho_{B C D}$. Because the mixed three-tangle is a convex function, the parameter $\tau_{3}\left(\rho_{B C D}\right)$ is nondecreasing under this POVM. Therefore, when the decrease of $\tau_{4}$ is less than the increase of $\tau_{3}$, the residual correlation $M_{C}$ will not be monotone. Just as in the example in Sec. II A, the changes of the three- and four-qubit correlations are $\Delta \tau_{3}\left(\rho_{B C D}\right)=$ -0.1964 and $\Delta \tau_{4}=0.08127$, respectively, which results in $\Delta M_{C}=-0.1151$. It should be pointed out that, for quantum states that do not have three-qubit correlations under LOCC (like the cluster-class states $\left|\Psi^{(1)}\right\rangle$ and $\left|\Psi^{(3)}\right\rangle$ ), the residual correlation $M_{k}$ could be entanglement monotone.

In this paper, we prove analytically that $E_{m s}$ is entanglement monotone for the four-qubit cluster-class states, and thus it can characterize the multipartite entanglement in the system. For general four-qubit states, $E_{m s}$ is conjectured to be entanglement monotone according to the numerical analysis in Ref. [9]. Moreover, for a type of four-qubit state, numerical analysis of Bell inequalities [28,29] shows a similar property to that of $E_{m s}$, which also supports our conjecture. A proof or disproof for an arbitrary $N$-qubit case is still awaited. At present, we know that, in a kind of quantum state whose two-qubit concurrences are zeros under the POVMs, the average correlation $E_{m s}=\Sigma_{k} \tau_{k\left(R_{k}\right)} / N$ is entanglement monotone. A trivial example is the $N$-qubit GHZ-class state $|\mathcal{G}\rangle_{N}=a|00 \ldots 0\rangle_{N}+b|11 \ldots 1\rangle_{N}$. A nontrivial example is a type of six-qubit cluster-class state $\left|\Psi_{6}\right\rangle=a|000000\rangle+b|000111\rangle$ $+c|111000\rangle-d|111111\rangle$, where the parameters $a, b, c$, and $d$ are complex numbers; the corresponding cluster states have been prepared recently by $\mathrm{Lu}$ et al. with a photon system [14].

In the four-qubit cluster-class states, the mixed threetangle $\tau_{3}$ is shown to be a compatible measure for quantifying the correlation $t_{3}$ in the QCRs. With this evaluation, the genuine four-qubit entanglement measure $\tau_{4}$ can be obtained. Based on this pure cluster state entanglement, we are able to introduce a mixed state entanglement measure by the convex roof extension [30], 


$$
\tau_{4}\left(\rho_{A B C D}\right)=\min \sum_{\left\{p_{x}, \phi_{x}^{(\mathcal{C})}\right\}} p_{x} \tau_{4}\left(\phi_{x}^{(\mathcal{C})}\right)
$$

where an extra restriction is that the general vector $\left|\phi_{x}^{(\mathcal{C})}\right\rangle$ in the pure state decomposition has the form of cluster-class states. As an example, we analyze the quantum state $\rho_{A B C D}=1 / 2\left(\left|\psi_{1}\right\rangle\left\langle\psi_{1}|+| \psi_{2}\right\rangle\left\langle\psi_{2}\right|\right)$, in which $\left|\psi_{1}\right\rangle=(|0000\rangle$ $+|1111\rangle) / \sqrt{2}$ and $\left|\psi_{2}\right\rangle=(|0011\rangle+|1100\rangle) / \sqrt{2}$. The general decomposition vector $\left|Z\left(q_{k}, \varphi_{k}\right)\right\rangle=\left(\sqrt{q_{k}}\left|\psi_{1}\right\rangle-e^{i \varphi_{k}} \sqrt{1-q_{k}}\left|\psi_{2}\right\rangle\right)$ has the form of the cluster-class state $\left|\Psi^{(1)}\right\rangle$. After choosing $q_{1}$ $=q_{2}=0.5, \varphi_{1}=0$, and $\varphi_{2}=\pi$, we can obtain $\tau_{4}\left(\rho_{A B C D}\right)=0$ in terms of the formula in Eq. (17). Furthermore, via the mixed state parameter $\tau_{4}$, one can solve the five-qubit correlation $t_{5}$ with the help of the QCRs, which can possibly be entanglement monotone in a kind of five-qubit pure state.
In conclusion, we have explored the multipartite quantum correlations in four-qubit cluster-class states. It is shown that the average multipartite correlation $E_{m s}$ is entanglement monotone in these systems, partly supporting our previous conjecture [9]. Moreover, we find a set of hierarchy measures $\left\{\tau_{2}, \tau_{3}, \tau_{4}\right\}$ that can characterize the different levels of entanglement in the cluster-class states. The entanglement monotone property of $E_{m s}$ in a general $N$-qubit pure state is still an open problem, which is worth study in the future.

\section{ACKNOWLEDGMENTS}

The authors would like to thank Dong Yang and Heng Fan for many useful discussions and suggestions. The work was supported by the RGC of Hong Kong under HKU Grants No. 7051/06P, No. 7012/06P, and No. 3/05C, the URC fund of HKU, and NSF-China Grant No. 10429401.
[1] A. K. Ekert, Phys. Rev. Lett. 67, 661 (1991).

[2] C. H. Bennett, G. Brassard, C. Crépeau, R. Jozsa, A. Peres, and W. K. Wootters, Phys. Rev. Lett. 70, 1895 (1993).

[3] C. H. Bennett and D. P. Divincenzo, Nature (London) 404, 247 (2000).

[4] R. Raussendorf and H. J. Briegel, Phys. Rev. Lett. 86, 5188 (2001).

[5] S.-S. Li, G.-L. Long, F.-S. Bai, S.-L. Feng, and H.-Z. Zheng, Proc. Natl. Acad. Sci. U.S.A. 98(21), 11847 (2001).

[6] R. Horodecki, P. Horodecki, M. Horodecki, and K. Horodecki, e-print arXiv:quant-ph/0702225; M. B. Plenio and S. Virmani, Int. J. Quantum Inf. 7, 1 (2007)

[7] V. Vedral, M. B. Plenio, M. A. Rippin, and P. L. Knight, Phys. Rev. Lett. 78, 2275 (1997).

[8] M. Jakob and J. A. Bergou, e-print arXiv:quant-ph/0302075; X. Peng, X. Zhu, D. Suter, J. Du, M. Liu, and K. Gao, Phys. Rev. A 72, 052109 (2005); T. E. Tessier, Found. Phys. Lett. 18, 107 (2005).

[9] Y.-K. Bai, D. Yang, and Z. D. Wang, Phys. Rev. A 76, 022336 (2007).

[10] D. Schlingemann and R. F. Werner, Phys. Rev. A 65, 012308 (2001).

[11] O. Gühne, G. Tóth, P. Hyllus, and H. J. Briegel, Phys. Rev. Lett. 95, 120405 (2005).

[12] P. Walther, K. J. Resch, T. Rudolph, E. Schenck, H. Weinfurter, V. Vedral, M. Aspelmeyer, and A. Zeilinger, Nature (London) 434, 169 (2005); R. Prevedel, P. Walther, F. Tiefenbacher, P. Böhi, R. Kaltenbaek, T. Jennewein, and A. Zeilinger, ibid. 445, 65 (2007).

[13] N. Kiesel, C. Schmid, U. Weber, G. Tóth, O. Gühne, R. Ursin, and H. Weinfurter, Phys. Rev. Lett. 95, 210502 (2005); G. Vallone, E. Pomarico, P. Mataloni, F. De Martini, and V. Berardi, ibid. 98, 180502 (2007); K. Chen, C.-M. Li, Q. Zhang, Y.-A. Chen, A. Goebel, S. Chen, A. Mair, and J.-W. Pan, ibid. 99, 120503 (2007).
[14] C.-Y. Lu, X.-Q. Zhou, O. Gühne, W.-B. Gao, J. Zhang, Z.-S. Yuan, A. Goebel, T. Yang, and J.-W. Pan, Nat. Phys. 3, 91 (2007)

[15] C. H. Bennett, S. Popescu, D. Rohrlich, J. A. Smolin, and A. V. Thapliyal, Phys. Rev. A 63, 012307 (2000).

[16] W. Dür, G. Vidal, and J. I. Cirac, Phys. Rev. A 62, 062314 (2000).

[17] J.-M. Cai, Z.-W. Zhou, X.-X. Zhou, and G.-C. Guo, Phys. Rev. A 74, 042338 (2006).

[18] Although the parameters $a, b, c$, and $d$ are real in the general case, we can enlarge them into complex numbers without loss of generality.

[19] S. Hill and W. K. Wootters, Phys. Rev. Lett. 78, 5022 (1997); W. K. Wootters, ibid. 80, 2245 (1998).

[20] E. Santos and M. Ferrero, Phys. Rev. A 62, 024101 (2000).

[21] F. Verstraete, J. Dehaene, and B. DeMoor, Phys. Rev. A 64, 010101(R) (2001).

[22] After the POVM $\left\{A_{1}, A_{2}\right\}$, the output states of the subsystem $\rho_{B}$ will be $\rho_{B}^{i}=\operatorname{Tr}_{A C D}\left(A_{i}\left|\Psi^{(2)}\right\rangle\left\langle\Psi^{(2)}\right| A_{i}^{\dagger}\right) / p_{i}$, for $i=1,2$, for which the relation $\rho_{B}=p_{1} \rho_{B}^{1}+p_{2} \rho_{B}^{2}$ holds. The cases for $\rho_{C}$ and $\rho_{D}$ are similar.

[23] T. J. Osborne and F. Verstraete, Phys. Rev. Lett. 96, 220503 (2006).

[24] A. Wong and N. Christensen, Phys. Rev. A 63, 044301 (2001).

[25] V. Coffman, J. Kundu, and W. K. Wootters, Phys. Rev. A 61, 052306 (2000).

[26] F. Verstraete, J. Dehaene, B. De Moor, and H. Verschelde, Phys. Rev. A 65, 052112 (2002).

[27] R. Lohmayer, A. Osterloh, J. Siewert, and A. Uhlmann, Phys. Rev. Lett. 97, 260502 (2006).

[28] S. Yu, Z.-B. Chen, J.-W. Pan, and Y.-D. Zhang, Phys. Rev. Lett. 90, 080401 (2003).

[29] J. Endrejat and H. Büttner, Phys. Rev. A 71, 012305 (2005).

[30] A. Uhlmann, Phys. Rev. A 62, 032307 (2000). 